\title{
Anisotropic decoherence in quantum wells with arbitrary magnetic fields: Interplay of the spin-orbit coupling terms
}

\author{
M. Prada ${ }^{1}$, and D. Pfannkuche ${ }^{1}$ \\ ${ }^{1}$ I. Institut für Theoretische Physik, Universität Hamburg, Jungiusstr. 9, 20355 Hamburg, Germany
}

(Dated: July 5, 2021)

\begin{abstract}
We present a theoretical study of the anisotropy of the spin relaxation and decoherence in typical quantum wells with an arbitrary magnetic field. In such systems, the orientation of the magnetic field relative to the main crystallographic directions is crucial, owing to the lack of spin-rotation symmetry. For typical high mobility samples, relaxation anisotropies owing to the interplay of Rashba and Dresselhaus spin orbit coupling are calculated. We also include the effect of the cubic-in-momentum terms. Although commonly ignored in literature, the latter were experimentally evidenced by the observation of strong anisotropy in spin decoherence measurements by different experimental groups and has long remained unexplained. This work suggests a method to determine the relative strength of spin-orbit coupling terms by angular resolution of decoherence in ESR experiments.
\end{abstract}

\section{INTRODUCTION}

Spin relaxation processes in semiconductors continue to attract attention in connection with a large number of spintronic applications [1-5], in which silicon appears to be a very suitable material due to long decoherence times [6] and high gate fidelity [10,-12]. Electron spin resonance (ESR) is a promising technique to manipulate spins directly in highmobility quantum well heterostructures (QW) [13-15]. In these systems, the main source of decoherence is typically spin-orbit coupling (SOC) due to bulk (BIA), structure (SIA) or interface inversion asymmetry (IIA) [16-18]. The interplay of the different SOC contributions may result in strong anisotropy of spin relaxation and decoherence, giving a hint on their relative strength.

In this work we describe decoherence and relaxation processes in general 2-DEG ESR experiments, quantifying the anisotropies in terms of the relative direction of external fields and main crystallographic directions. The appropriate choice of coordinate system is crucial in the calculations of spinrelated observables, owing to the anisotropy that follows the interplay of the different contributions of SOC. Scattering relaxation events and SOC are defined within the 2-DEG perpendicular to the growth direction $\hat{k}$, determining the crystallographic coordinate system $\{\hat{i}, \hat{j}, \hat{k}\}$ (black arrows of Fig. 1), whereas the quantization axis is naturally determined by the direction of the magnetic field $\hat{z}$. The magnetic coordinate system $\{\hat{x}, \hat{y}, \hat{z}\}$ (blue arrows of Fig. 1), is related to the crystallographic one by the Euler-Rodrigues formula, consisting of a rotation along $\hat{n}$ by the polar angle $\theta$ (see Fig. 1). During an ESR experiment, the magnetic field direction is changed relative to the crystallographic axis, such that $\theta$ varies while the azimuth $\varphi$ remains constant. For $\theta=0$, spin-rotation symmetry $\mathrm{SU}(2)$ is completely broken (reduced to $\mathrm{U}(1)$ ), if both BIA and SIA (only BIA or only SIA) SOC are present [19]. As $\theta$ is varied, spin-rotation symmetry is completely broken, and a measurement of the anisotropy in spin observables is then expected to be captured in terms of the azimuthal angle.

\footnotetext{
mprada@physnet.uni-hamburg.de
}

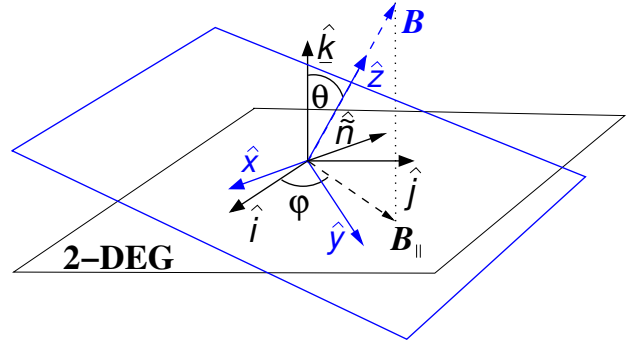

FIG. 1. The main quantization axis $\hat{z}$ is given by $\boldsymbol{B}$, determining the magnetic coordinate system (blue), with $\hat{x}, \hat{y}$ being arbitrary directions in the plane perpendicular to $\hat{z}$. The crystallographic coordinate system typically define the electronic motion $(\hat{i}, \hat{j})$ and the growth direction $(\hat{k})$ (black). Any (pseudo) vector can be expressed in either coordinate system, with its components being related by the Euler-Rodrigues rotation matrix, $R_{\hat{n}}(\theta)$.

As a result, the relative strength of the different SOC contributions can be experimentally determined in angle-resolved measurements.

The aim of this work is to achieve a complete description of D'yakonov-Perel' decoherence and relaxation processes as a function of the main angles, capturing the effects of the anisotropy that results from the interplay of BIA and SIA. We find that the choice of the magnetic coordinate system possesses clear advantages, rendering Redfield-type equations of motion with analytical solutions. We also include the effects of the commonly neglected cubic-in-momentum terms, which may become important in typical experiments. Our theoretical model finds an excellent agreement with experimental data on high-mobility QWs, where the existence of strong anisotropies have long remained unexplained.

This article is organized as follows: in Section II, we describe spin relaxation and decoherence processes. First, (II A) we define the SOC in the magnetic coordinate system, then we write the equations of motion within the Redfield approach (II B), and finally, we obtain an expression for $T_{1}$ and $T_{2}$ (IIC), first for a perpendicular magnetic field with linear SOC terms, then for magnetic fields with arbitrary direction and last, including the effects of cubic in momentum SOC terms. Section III is devoted to results, from a theoretical perspective (III A) and in connection with experiments (IIIB). We finally give some concluding remarks in section IV 


\section{METHODS}

\section{A. SOC terms in magnetic coordinates}

We consider a general 2-DEG sample with an external magnetic field $\boldsymbol{B}$ whose direction is defined by the polar angle $\theta$ and the azimuth $\varphi$. The latter corresponds to angle of the main crystallographic direction $\hat{i}$ and the projection of the magnetic field onto the 2-DEG (see Fig. 1). The direction of the magnetic field $\boldsymbol{B}=B \sigma_{z}$ determines the magnetic coordinate system, $\hat{x}, \hat{y}, \hat{z}$ and the quantization of the spin, $\sigma_{z}$,

$$
H_{0}=-\frac{1}{2} g \mu_{B} B \sigma_{z}
$$

Here, $\mu_{B}$ is the Bohr magneton and $g$ denotes the $g$-factor, giving a Larmor frequency of precession $\omega_{l} \simeq g \mu_{B} B / \hbar$.

Rashba- (SIA) and Dresselhaus- (BIA) type coupling terms appear in the Hamiltonian, owing to the motion of electrons confined to the 2DEG with broken inversion symmetry. Note that BIA terms are not limited to systems without bulk inversion (as GaAs, for instance) but also in two-dimensional centrosymmetric-based materials such as $\mathrm{Si} / \mathrm{SiGe}$, owing to interface-inversion asymmetry [21, 22]. Moreover, the strength of the BIA- related coupling parameter has been determined for the latter, yielding a larger value than the SIA coupling [20]. To describe SOC, it is customary to choose the Hilbert space spanned by the Pauli matrices $\left\{\tilde{\sigma}_{l}\right\}$ along the crystallographic directions, $l=i, j, k$, in which the SOC contributions to lowest order in momentum reads:

$$
H_{1}=\alpha(\tilde{\boldsymbol{\sigma}} \times \tilde{\boldsymbol{k}}) \cdot \hat{k}+\beta\left(\tilde{\boldsymbol{\sigma}}_{i} \tilde{k}_{i}-\tilde{\boldsymbol{\sigma}}_{j} \tilde{k}_{j}\right) .
$$

Here $\alpha, \beta$ determine the strength of the Rashba and Dresselhaus coupling to lowest order in $k$. Symbol $\sim$ over $o(\tilde{o})$ denotes that $o$ is expressed in the crystallographic coordinate system.

Higher order in momentum terms may be added [23],

$$
H_{\mathrm{c}}=\beta_{3}\left(\tilde{\sigma}_{j} \tilde{k}_{j} \tilde{k}_{i}^{2}-\tilde{\sigma}_{i} \tilde{k}_{i} \tilde{k}_{j}^{2}\right)+\alpha_{3}\left(\tilde{\sigma}_{i} \tilde{k}_{j} \tilde{k}_{i}^{2}-\tilde{\sigma}_{j} \tilde{k}_{i} \tilde{k}_{j}^{2}\right),
$$

with $\alpha_{3}, \beta_{3}$ being the cubic Rashba and Dresselhaus coupling to third order in momentum. Bearing in mind that $\beta$ is proportional to $\left\langle k_{k}^{2}\right\rangle \propto l^{-1}$ with $l$ being the well width, we note that the cubic terms may become important with respect to the linear Dresselhaus one as $l$ increases. The cubic terms are also enhanced as the carrier density increases, bearing a larger in-plane $k_{i}, k_{j}[36]$.

Scattering relaxation occurs as the $\vec{k}$ vector changes randomly direction while the modulus remain constant, $|\vec{k}| \simeq k_{F}$, with $k_{F}$ being the Fermi wavevector. Classically and neglecting scattering events, the circular motion of the electron is given by the cyclotron frequency, $\omega_{c}(\theta)=e B \cos \theta / m$, setting $\tilde{\boldsymbol{k}} \simeq k_{F}\left(\cos \omega_{c} t \hat{i}+\sin \omega_{c} t \hat{j}\right)$. In addition, elastic scattering events change randomly the momentum direction in a mean interval $\tau_{k}$. Due to this cyclotron motion and random scattering, the electron 'feels' an average SOC fluctuating field which typically is smaller than its instantaneous local value, commonly termed as 'motional narrowing' [24, 25] within the
D'yakonov-Perel' (DP) limit [26, 27]. Hence, it appears convenient to express the SOC in terms (2) of an effective fluctuating magnetic field in the magnetic coordinate system by a transformation defined in Appendix A, namely, an EulerRodrigues rotation of all the (pseudo-) vectors in Eq. (2), $\tilde{\boldsymbol{\sigma}}=R_{\hat{\tilde{n}}}^{\theta} \boldsymbol{\sigma} ; \tilde{\boldsymbol{k}}=R_{\hat{\tilde{n}}}^{\theta} \boldsymbol{k}, R_{\hat{\tilde{n}}}^{\theta}$ being the rotation matrix of an angle $\theta$ along $\hat{\tilde{n}}$, as depicted in Fig 1 In this rotated frame the time dependency factorizes,

$$
H^{\prime}=H_{1}+H_{\mathrm{c}}=\vec{\sigma} \vec{B}^{\mathrm{eff}}, \quad \vec{B}^{\mathrm{eff}}=\operatorname{Re}\left\{e^{i \omega_{a} t} \vec{Z}_{a}\right\}
$$

with $a=\mathrm{R}, \mathrm{D}, 3 \mathrm{R}, 3 \mathrm{D}$ labeling Rashba, Dresselhaus or cubic SOC components, $\omega_{R}=-\omega_{D}=\omega_{c} \cos \theta$, and $\omega_{3 R}=-\omega_{3 D}=$ $3 \omega_{c} \cos \theta$ (see insets of Fig. 2). The components of the bidimensional pseudo-vector $Z_{a}$ along the magnetic axis are obtained in App. A and summarized in table $\mathrm{I}$. We note that in the presence of a magnetic field, the canonical momentum should be replaced by the kinetic one, $\boldsymbol{k} \rightarrow \boldsymbol{k}-\boldsymbol{e} \boldsymbol{A}$, with $\boldsymbol{A}$ being the vector potential. However, the additional term is neglectible in typical laboratory frames in high mobility samples. Moreover, terms in $\boldsymbol{A}$ would not contribute to the anisotropy in $\varphi$.

\section{B. Equations of motion}

In order to evaluate spin relaxation and decoherence, we solve the master equations for the density matrix in the interaction representation,

$$
\rho^{*}(t)=\frac{1}{2}\left[1+\vec{n}(t) \vec{\sigma}^{*}(t)\right]
$$

with

$$
o^{*}(t)=e^{i H_{0} t} o(t) e^{-i H_{0} t}, \quad o=\rho, H^{\prime}, \sigma
$$

We stress that $\rho^{*}$ is represented in the magnetic coordinate system. This choice is crucial, as it determines the correct quantization axis, which coincides with the direction of $\boldsymbol{B}$ as long the Zeeman energy is large compared with the spinorbit coupling, as occurs in typical ESR experiments. We now derivate (5) to get:

$$
\begin{aligned}
\frac{\mathrm{d}\left[n_{i}(t) \sigma^{*}(t)\right]}{\mathrm{d} t} & =\frac{\mathrm{d} n_{i}(t)}{\mathrm{d} t} \sigma^{*}(t)+\frac{i}{\hbar} n_{i}(t) H_{0} e^{i H_{0} t / \hbar} \sigma_{i}(t) e^{-i H_{0} t / \hbar}- \\
& -\frac{i}{\hbar} n_{i}(t) e^{i H_{0} t / \hbar} \sigma_{i}(t) H_{0} e^{-i H_{0} t / \hbar}
\end{aligned}
$$

giving:

$$
\frac{\mathrm{d} n_{i}(t)}{\mathrm{d} t} \sigma^{*}(t)=-\frac{i}{\hbar} n_{i}(t)\left[H_{0}, \sigma_{i}^{*}(t)\right]+\frac{\mathrm{d}\left[n_{i}(t) \sigma^{*}(t)\right]}{\mathrm{d} t}
$$

On the other hand, we have, to second order:

$$
\frac{\mathrm{d} \rho^{*}}{\mathrm{~d} t} \simeq \frac{i}{\hbar}\left[\rho_{0}^{*}, H_{\mathrm{SOC}}^{*}(t)\right]+\left(\frac{i}{\hbar}\right)^{2} \int_{0}^{t}\left[\left[\rho_{0}^{*}, H_{\mathrm{SOC}}^{*}\left(t^{\prime}\right)\right], H_{\mathrm{SOC}}^{*}(t)\right] \mathrm{d} t^{\prime}
$$


TABLE I. Components of the effective Rashba $(a=R)$ and Dresselhaus $(a=D, 3 D)$ effective magnetic field along the magnetic axis, $q=x, y, z$.

\begin{tabular}{|c||c|c|}
\hline$Z_{a}^{q}$ & $a=\mathrm{R}(3 \mathrm{R})$, prefactor $\alpha k_{F}\left(\alpha_{3}\left(k_{F}^{3}\right)\right)$ & $a=\mathrm{D}(3 \mathrm{D})$, prefactor $\beta k_{F}\left(\beta_{3}\left(k_{F}^{3}\right)\right)$ \\
\hline \hline$q=x$ & $(1-\cos \theta) \sin \varphi \cos \varphi-i\left[\cos \theta \cos ^{2} \varphi+\sin ^{2} \varphi\right]$ & $1-\cos ^{2} \varphi(1-\cos \theta)-i(1-\cos \theta) \sin \varphi \cos \varphi$ \\
\hline$q=y$ & $\cos \theta \sin ^{2} \varphi+\cos ^{2} \varphi+i(1-\cos \theta) \sin \varphi \cos \varphi$ & $(1-\cos \theta) \sin \varphi \cos \varphi+i\left[1-\sin ^{2} \varphi(1-\cos \theta)\right]$ \\
\hline$q=z$ & $\sin \theta(\sin \varphi+i \cos \varphi)$ & $-\sin \theta(\cos \varphi-i \sin \varphi)$ \\
\hline
\end{tabular}

with $\rho_{0}^{*}=\rho^{*}(t=0)$. Using (4) and (7), we obtain:

$$
\begin{aligned}
& \frac{\mathrm{d}\left[n_{i}(t) \sigma_{i}^{*}(t)\right]}{\mathrm{d} t} \simeq \frac{i}{\hbar} n_{i}(0)\left[\sigma_{i}^{*}(0), \sigma_{q}^{*}(t)\right] \overline{B_{q}^{\mathrm{eff}}}+ \\
& \quad+\left(\frac{i}{\hbar}\right)^{2} \int_{0}^{t} n_{i}(0)\left[\left[\sigma_{i}^{*}(0), \sigma_{q}^{*}\left(t^{\prime}\right)\right], \sigma_{q^{\prime}}^{*}(t)\right] \mathcal{B}_{q q^{\prime}}\left(t, t^{\prime}\right) \mathrm{d} t^{\prime}
\end{aligned}
$$

with the auto-correlation function, $\mathcal{B}_{q q^{\prime}}\left(t, t^{\prime}\right)$ defined as:

$$
\mathcal{B}_{q q^{\prime}}\left(t, t^{\prime}\right) \equiv \overline{B_{q}^{\text {eff }}(t) B_{q^{\prime}}^{\text {eff }}\left(t^{\prime}\right)}
$$

We consider in the integral of the right hand of Eq. (8) ensembles of systems with equal $H_{0}, \rho_{0}$, but with the effective SOC field varying from sample to sample due to different scattering configurations. Hence, for our stationary perturbation $H^{\prime}$, this ensemble average depends only on the interval $\tau=t-t^{\prime}$.

On what follows, we make the following assumptions: (i) we may use the Redfield approximation [28], replacing $\rho(0)[\sigma(0)]$ by $\rho(t)[\sigma(t)]$ in the integrals, such that the evolution equation depends only on the present state, (ii) we may use the Markovian approximation, extending the upper limit of the integral to infinity, (iii) we may neglect the correlation between $H^{\prime}$ and $\rho^{*}(0)$. (i) - (iii) are justified as long as $\overline{\left|H^{\prime}\right|^{2}} \sim\left(H^{\prime}(0)\right)^{2} e^{-t / \tau_{k}} \ll \hbar^{2} \tau_{k}^{-1} t^{-1}$, for times $t$ beyond the transient limit, $t \gg \tau_{k}$. Here, we assumed an exponentially decreasing auto-correlation function for the fluctuating spinorbit field, with the time constant given by the scattering time $\tau_{k}$. Combining Eqs. (7, 9) and the expression in (1):

$$
\begin{aligned}
& \frac{\mathrm{d} n_{i}(t)}{\mathrm{d} t} \sigma_{i}^{*}(t)=-\frac{i}{\hbar} n_{i}(t) B_{0}\left[\sigma_{z}^{*}, \sigma_{i}^{*}\right]- \\
& -\frac{1}{\hbar^{2}} \int_{0}^{\infty} n_{i}(t)\left[\left[\sigma_{i}^{*}(t), \sigma_{q}^{*}(t-\tau)\right], \sigma_{q^{\prime}}^{*}(t)\right] \mathcal{B}_{q q^{\prime}}(\tau) \mathrm{d} \tau
\end{aligned}
$$

Multiplying Eq. (10) by $\sigma_{r}^{*}$, taking the trace, and using the identities:

$$
\operatorname{Tr}\left\{\sigma_{i}^{*} \sigma_{j}^{*}\right\}=2 \delta_{i j}, \quad\left[\sigma_{i}^{*}, \sigma_{j}^{*}\right]=2 i \varepsilon_{i j k} \sigma_{k}^{*},
$$

with $\varepsilon_{i j k}$ being the Levi-Civita symbol, we obtain:

$$
\begin{aligned}
& \frac{\mathrm{d} n_{i}(t)}{\mathrm{d} t} 2 \delta_{i r}=-\frac{2 i^{2} B_{0}}{\hbar} \varepsilon_{z i p}\left(2 \delta_{p r}\right) n_{i}(t)- \\
& -\left(\frac{i}{\hbar}\right)^{2} \operatorname{Tr}\left\{\int_{0}^{\infty}\left[\left[\sigma_{i}^{*}(t), \sigma_{q}^{*}(t-\tau)\right], \sigma_{q^{\prime}}^{*}(t)\right] \sigma_{r}^{*}(t) \mathcal{B}_{q q^{\prime}}(\tau) \mathrm{d} \tau\right\}
\end{aligned}
$$

The integral of Eq. (11) is evaluated in appendix $\mathrm{B}$ resulting in

$$
\frac{2}{\hbar^{2}} n_{i}(t)\left(\delta_{q^{\prime} i} \delta_{r k}-\delta_{q^{\prime} k} \delta_{i r}\right)\left\langle\alpha\left|\sigma_{q}\right| \beta\right\rangle\left\langle\beta\left|\sigma_{k}\right| \alpha\right\rangle b_{q q^{\prime}}\left(\omega_{l}\right)
$$

with $b_{q q^{\prime}}$ being the Fourier transform of the autocorrelation function,

$$
b_{q q^{\prime}}(\omega)=\int_{0}^{\infty} \mathcal{B}_{q q^{\prime}}(\tau) e^{-i \omega \tau} \mathrm{d} \tau
$$

Eq. (11) describes then a linear evolution of the elements of the density matrix,

$$
\dot{\vec{n}}=-A \vec{n}
$$

with

$A=\left(\begin{array}{ccc}b_{y y}\left(\omega_{l}\right)+b_{z z}(0) & B_{0} \hbar-b_{x y}\left(\omega_{l}\right) & -b_{x z}\left(\omega_{l}\right) \\ B_{0} \hbar-b_{x y}\left(\omega_{l}\right) & b_{x x}\left(\omega_{l}\right)+b_{z z}(0) & -b_{y z}\left(\omega_{l}\right) \\ -b_{z x}(0) & -b_{z y}(0) & b_{x x}\left(\omega_{l}\right)+b_{y y}\left(\omega_{l}\right) .\end{array}\right)$

In general, the eigenvalues of $A$ will consist of a complex conjugate pair and a real number. $T_{1}^{-1}$ corresponds to the real one and $T_{2}^{-1}$ to the real part of the conjugate pair. In this sense, we may disregard the first term on the right of Eq. (11), which would only contribute to the imaginary part of the eigenvalues of (14).

Eq. (14) corresponds to a general result within Redfield's approach [24, 25] that describes the time evolution of the spin components, which ultimately yields dephasing and relaxation spin times. In the next sections, we employ this approach to derive $T_{1}^{-1}$ and $T_{2}^{-1}$ in experimentally relevant systems.

\section{Decoherence and relaxation in typical ESR experiments}

We evaluate next the autocorrelation functions $\mathcal{B}_{q q^{\prime}}(\tau)$ and its Fourier transform, $b_{q q^{\prime}}$ in typical samples of our interest, with effective fields given by (3). On these systems, we can assume that $\mathcal{B}_{q q^{\prime}}(\tau)$ is an even function of time, such that $b_{q q^{\prime}}(\omega)=b_{q q^{\prime}}(-\omega)$, and that the $x$-, $y$ - and $z$-components of the field fluctuate independently. Noting that the time dependency of $B_{q}^{\text {eff }}$ is determined by the scattering processes and the cyclotron motion, we can write, for the linear contributions: 


$$
\mathcal{B}_{q q^{\prime}}(\tau) \simeq \overline{\left(Z_{q, D} e^{i \omega_{c} \tau / 2}+Z_{q, R} e^{-i \omega_{c} \tau / 2}\right)\left(Z_{q^{\prime}, D}^{*} e^{i \omega_{c} \tau / 2}+Z_{q^{\prime}, R}^{*} e^{-i \omega_{c} \tau / 2}\right)} e^{-|\tau| / \tau_{k}}
$$

leading to:

$$
\begin{array}{r}
\mathcal{B}_{q q^{\prime}}(\tau) \simeq\left[Z_{q, D} Z_{q^{\prime}, D}^{*} e^{i \omega_{c} \tau}+Z_{q, D} Z_{q^{\prime}, R}^{*}+Z_{q, R} Z_{q^{\prime}, D}^{*}\right. \\
\left.+Z_{q, R} Z_{q^{\prime}, R}^{*} e^{-i \omega_{c} \tau}\right] e^{-|\tau| / \tau_{k}},
\end{array}
$$

and the Fourier transform,

$$
\begin{aligned}
b_{q q^{\prime}} \simeq \quad & \left(\frac{Z_{q, D} Z_{q^{\prime}, D}^{*} \tau_{k}}{1+\left(\Omega_{-} \tau_{k}\right)^{2}}+\frac{Z_{q, R} Z_{q^{\prime}, R}^{*} \tau_{k}}{1+\left(\Omega_{+} \tau_{k}\right)^{2}}+\right. \\
+ & \left.+\frac{\left[Z_{q, D} Z_{q^{\prime}, R}^{*}+Z_{q, R} Z_{q^{\prime}, D}^{*}\right] \tau_{k}}{1+\left(\omega_{l} \tau_{k}\right)^{2}}\right) \delta_{q q^{\prime}}
\end{aligned}
$$

with $\Omega_{ \pm}=\omega_{l} \pm \omega_{c}$. We stress that the different terms for the auto-correlation function are not present in other approaches [29], and as we will see, may boost the anisotropy on the decoherence in some cases.

\section{Perpendicular magnetic fields}

We first focus on the most straight-forward case, where the external magnetic field is parallel to the growth direction $\hat{k}$, $\theta=0$. The crystallographic and magnetic coordinate systems coincide and hence, fluctuations in the effective magnetic field occur only along $\hat{x}$ - and $\hat{y}$-directions $\left(b_{z z}(\omega)=0\right)$, preserving the $\mathrm{U}(1)$ symmetry: $Z_{x, R}(\theta=0)=-i \alpha k_{F}, Z_{x, D}(\theta=0)=\alpha k_{F}$, $Z_{y, R}(\theta=0)=\beta k_{F}$ and $Z_{y, D}(\theta=0)=i \beta k_{F}$, with the remaining $Z_{q, R / D}$ being 0 , bearing $b_{x y}(\omega)=b_{x z}(\omega)=b_{z z}(\omega)=0$. We obtain:

$$
b_{x x}\left(\omega_{l}\right)=b_{y y}\left(\omega_{l}\right)=k_{F}^{2} \tau_{k}\left(\frac{\beta^{2}}{1+\left(\Omega_{-} \tau_{k}\right)^{2}}+\frac{\alpha^{2}}{1+\left(\Omega_{+} \tau_{k}\right)^{2}}\right) .
$$

Eq. (14) contains only diagonal terms, giving an straight forward expression for $T_{1,2}$ :

$$
T_{1}^{-1}(\theta=0)=\frac{8 \tau_{k} k_{F}^{2}}{\hbar^{2}}\left(\frac{\beta^{2}}{1+\left(\Omega_{-}^{0} \tau_{k}\right)^{2}}+\frac{\alpha^{2}}{1+\left(\Omega_{+}^{0} \tau_{k}\right)^{2}}\right)
$$

with $\Omega_{ \pm}^{0}=\omega_{c}^{0} \pm \omega_{l}, \omega_{c}^{0} \equiv \omega_{c}(\theta=0)$. This result reflects the additive contributions of spin relaxation due to Rashba and Dresselhaus. The Rashba (Dresselhaus) effective fields in the electronic frame fluctuate at $\Omega_{+}^{\theta}=\left(\omega_{l}+\omega_{c}^{0} \cos \theta\right)\left(\Omega_{-}^{\theta}=\right.$ $\left.\mid \omega_{l}-\omega_{c}^{0} \cos \theta\right) \mid$. As a result, $T_{1,2}^{-1}$ has a minimum as a function of $\tau_{k}$ when $\Omega_{-} \tau_{k}=1$. Viewing the problem from the rotating frame of the effective magnetic field (the one rotating at $\Omega_{-}=\omega_{l}-\omega_{c}$, which is the resulting rate of change of the effective magnetic field due to SOC), these results are reasonable, since $T_{1}$ corresponds to the time it takes to change the $z$-magnetization. Such change is brought about by 'static' fields in either the $x$ - or $y$-directions in the rotating frame. But 'static' $x$ - or $y$-fields in the rotating frame oscillate at $\Omega_{-}$in the laboratory frame. If the scattering time $\tau_{k}$ is comparable to $\Omega_{-}^{-1}$, then the $T_{1}$ process become most effective, as the electron has time to 'feel' the effects of the change in $x$ - or $y$ fields. In the rapid motion limit, $\Omega_{-} \tau_{k}, \omega_{c} \tau_{k} \ll 1$, we note that $T_{i}^{-1} \propto \tau_{k}$, i.e., the shorter $\tau_{k}$ (that is, the more rapid the motion), the narrower the resonance. This phenomenon is therefore called motional narrowing: The motion narrows the resonance because it allows a given spin to sample many fields, some of which cause it to advance in phase; others, to be retarded. The dephasing takes place, then, by a random walk of small steps, each one much less than a radian. It is well known that in this limit, $T_{2}^{-1}(0) \simeq T_{1}^{-1}(0) / 2$, due to the absence of fluctuations of the effective field along $\hat{z}$.

In contrast, when there is no 'motion', a given spin experiences a constant local field. Each collision gives a loss in phase memory, and thus a more rapid collision rate produces a shorter phase memory and a broader line, thus termed as 'collision broadening'. $T_{2}^{-1}$ would then be proportional to the collision rate, $\tau_{k}^{-1}$, which is in clear contrast with the motional narrowing: the phase of the oscillation is then changed by each collision. Clearly, for $\omega_{c}=\omega_{l}$, the SOC field appears static, and the DP model breaks down. On typical experimental setups, we have that $\omega_{c}(0) \gg \omega_{l}$, however, as $\theta \simeq \pi / 2$, the collision broadening limit can be reached, as we will see.

\section{Arbitrary magnetic fields}

As the external magnetic field $\boldsymbol{B}$ is tilted with respect to the crystallographic axis, the projections of the electronic movement in $\hat{i}, \hat{j}$ onto $\hat{z}$ result in effective fluctuating fields along $z$-axis, resulting in anisotropic contributions:

$$
T_{2}^{-1} \simeq \frac{2}{\hbar^{2}}\left(b_{x x}\left(\omega_{l}\right)+b_{y y}\left(\omega_{l}\right)+2 b_{z z}(0)\right),
$$

where we have assumed that the components of the effective field fluctuate independently, $b_{\alpha \alpha^{\prime}}=0, \alpha \neq \alpha^{\prime}$. Using Eqs. 97, (13) and table I, we have:

$$
b_{z z}(0)=\tau_{k} \frac{k_{F}^{2} \sin ^{2} \theta\left(\alpha^{2}+\beta^{2}+\alpha \beta \sin 2 \varphi\right)}{1+\omega_{c}^{2} \tau_{k}^{2} \cos ^{2} \theta} .
$$

$b_{z z}(0)$ is maximal along the [110] direction $\left(\varphi=45^{\circ}\right)$, where both contributions of SOC are parallel, and minimal along [110], $\left(\varphi=-45^{\circ}\right)$ where these are anti-parallel (see inset of fig. 22. Note that the fluctuations occur with a frequency given by $\omega_{c}$ in the electronic frame. The other two terms can be 
combined to give:

$$
\begin{aligned}
b_{x x}\left(\omega_{l}\right)+b_{y y}\left(\omega_{l}\right)= & 2 \tau_{k}\left(\frac{\left|Z_{D}^{x}\right|^{2}+\left|Z_{D}^{y}\right|^{2}}{1+\left(\Omega_{-}^{\theta} \tau_{k}\right)^{2}}+\frac{\left|Z_{R}^{x}\right|^{2}+\left|Z_{R}^{y}\right|^{2}}{1+\left(\Omega_{+}^{\theta} \tau_{k}\right)^{2}}\right. \\
& \left.+\frac{2 \operatorname{Re}\left\{Z_{D}^{x} Z_{R}^{x *}+Z_{R}^{y} Z_{D}^{y *}\right\}}{1+\left(\omega_{l} \tau_{k}\right)^{2}}\right) .
\end{aligned}
$$

The first two terms on the right appeared already in Eq. (17), pertaining to the fluctuations of the effective magnetic field along $x$ - and $y$ - axis. Looking at table If we find $\left|Z_{D(R)}^{x}\right|^{2}+$ $\left|Z_{D(R)}^{y}\right|^{2}=\beta^{2}\left(\alpha^{2}\right) k_{F}^{2}\left(1+\cos ^{2} \theta\right)$.

The last term of Eq. (19) reflects an interference effect, arising due to the correlations of the linear Rashba and Dresselhaus effective field, for which the cyclotron precession is canceled in the electronic frame. This highly anisotropic term $\left(\operatorname{Re}\left\{Z_{x, D} Z_{x, R}^{*}+Z_{y, R} Z_{y, D}^{*}\right\}=2 \alpha \beta \sin ^{2} \theta \cos 2 \varphi\right)$ results from the fluctuations of the projections of the SOC onto the $z$-axis, hence proportional to $\sin ^{2} \theta$. It has a maximal (minimal) value for $\varphi=\pi / 4(\varphi=-\pi / 4)$. Although a term with some similarities on the angular dependency was obtained by Glazov et al. [29], the overall expression differs substantially with our results, and also a connection with effective fluctuating fields or motional narrowing was not provided in [29].

The anisotropy can be quantified, in the linear SOC limit, in terms of a single parameter, $\chi=\beta / \alpha$, suggesting a method to determine the relative strength of both linear SIA and BIA couplings using ESR:

$$
\frac{T_{2}^{-1}(\theta, \varphi)}{T_{2}^{-1}(0)} \simeq \frac{1}{2 v}\left[\left(1+\cos ^{2} \theta\right)\left(\frac{1}{1+\left(\Omega_{+}^{\theta} \tau_{k}\right)^{2}}+\frac{\chi^{2}}{1+\left(\Omega_{-}^{\theta} \tau_{k}\right)^{2}}\right)+\sin ^{2} \theta\left(\frac{2 \chi \sin 2 \varphi}{1+\left(\omega_{l} \tau_{k}\right)^{2}}+\frac{2\left(1+\chi^{2}+\chi \sin 2 \varphi\right)}{1+\omega_{c}^{2} \tau_{k}^{2} \cos ^{2} \theta}\right)\right]
$$

with $v=\hbar^{2} T_{1}^{-1}(0) /\left(\tau_{k} k_{F}^{2} \alpha^{2}\right)$ and $T_{2}^{-1}(0)=T_{1}^{-1} / 2$.

\section{Decoherence including cubic SOC terms}

So far we did not take into account higher-in-momentum terms for the SOC. As it has been recently reported in liter- ature, cubic terms can be large compared to the linear terms in narrow QWs [30]. Even if these are small compared with the linear terms, interference effects due to simultaneous SOC sources are highly anisotropic. Moreover, the effective fields precess faster during the cyclotron motion of the electron, suggesting that a full derivation of the decoherence including all SOC is necessary. Defining the dimensionless relative strengths, $\Gamma_{\alpha}=\left\langle k_{i}^{2}\right\rangle \alpha_{3} / \alpha$ and $\Gamma_{\beta}=\left\langle k_{i}^{2}\right\rangle \beta_{3} / \alpha$, we get:

$$
\begin{aligned}
& \frac{T_{2}^{-1}(\theta, \varphi)}{T_{2}^{-1}(0)} \simeq \frac{1}{2 v}\left[\left(1+\cos ^{2} \theta\right)\left(\frac{1}{1+\left(\Omega_{+}^{\theta} \tau_{k}\right)^{2}}+\frac{\chi^{2}}{1+\left(\Omega_{-}^{\theta} \tau_{k}\right)^{2}}+\frac{\Gamma_{\alpha}}{1+\left(\Omega_{2+}^{\theta} \tau_{k}\right)^{2}}+\frac{2 \chi \Gamma_{\beta}}{1+\left(\Omega_{2-}^{\theta} \tau_{k}\right)^{2}}+\frac{\left(\Gamma_{\alpha}\right)^{2}}{1+\left(\Omega_{3+}^{\theta} \tau_{k}\right)^{2}}+\frac{\left(\Gamma_{\beta}\right)^{2}}{1+\left(\Omega_{3-}^{\theta} \tau_{k}\right)^{2}}\right)\right. \\
& \left.\quad+\sin ^{2} \theta\left(\frac{2\left(\chi+\Gamma_{\alpha} \Gamma_{\beta}\right) \sin 2 \varphi}{1+\left(\omega_{l} \tau_{k}\right)^{2}}+\frac{2 \Gamma_{\beta} \sin 2 \varphi}{1+\left(\Omega_{-}^{\theta} \tau_{k}\right)^{2}}+\frac{2 \chi \Gamma_{\alpha} \sin 2 \varphi}{1+\left(\Omega_{+}^{\theta} \tau_{k}\right)^{2}}+\frac{2\left(1+\chi^{2}+\chi \sin 2 \varphi\right)}{1+\omega_{c}^{2} \tau_{k}^{2} \cos ^{2} \theta}+\frac{2 \chi\left(\Gamma_{\alpha}+\Gamma_{\beta}\right)}{1+4 \omega_{c}^{2} \tau_{k}^{2} \cos ^{2} \theta}+\frac{\left(\Gamma_{\alpha}\right)^{2}+\left(\Gamma_{\beta}\right)^{2}}{1+9 \omega_{c}^{2} \tau_{k}^{2} \cos ^{2} \theta}\right)\right]
\end{aligned}
$$

with $\Omega_{n \pm}^{\theta}=n \omega_{c} \cos \theta \pm \omega_{l}, n=2,3$, and $v$ determined now by a relaxation time that includes cubic contributions. Previous work on the angular dependency of $T_{2}$ yielded a different expression (see Eqs. (10), (11) and (15) of Glazov et al. [29]), where a classical field approach for the kinetic equations within the density matrix formalisms resulted in $\chi$ and $\varphi$-independent expressions for $T_{2}$ without third harmonic contributions. Our results yield a more general theory, where the auto-correlations of the effective fluctuating fields are explicitly taken into account. As we will see below, the experimental data indicates that the cubic contributions as well as interference terms resulting from the field correlations are critical for an accurate description of decoherence rates.

\section{RESULTS}

\section{A. Theoretical results}

Figure 2 shows the angular dependency of the normalized decoherence, $T_{2}^{-1}(\theta) / T_{2}^{-1}(0)$, for linear SOC, $\chi=1.5 \gg \Gamma_{\alpha, \beta}$ (solid colored lines), moderate cubic SOC, $\chi=1.5 \sim 2 \Gamma_{\alpha, \beta}$ (thin green curve) and large cubic SOC, $\Gamma_{\alpha, \beta} \gg \chi$ (broken green curve). The black curve, for comparison, corresponds to $\chi=0$, hence spin-rotation symmetry is only partially broken down to U(1), and the angle $\varphi$ is irrelevant: The effective magnetic field has the same magnitude along any direction in the 2DEG (see Fig. 2a.). The results illustrate that $T_{2}$ processes are greatly suppressed in the presence of a perpendicular field, as the electron can circle around with many 

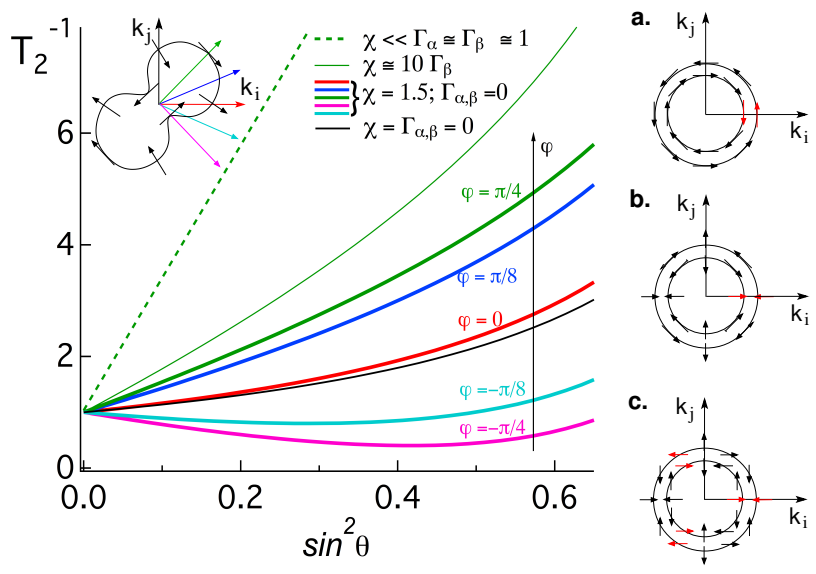

FIG. 2. $T_{2}^{-1} / T_{2}^{-1}(0)$ as a function $\sin ^{2} \theta, \theta=\measuredangle(\hat{k}, \hat{z})$, for five orientations of $B$ relative to the crystallographic axis, $\varphi=0, \pm \pi / 8, \pm \pi / 4$ in the linear SOC limit (thick solid lines), including moderate cubic terms (thin green curve), and large cubic terms (broken green trace)Left inset: magnitude (solid black curve) and direction (black arrows) of the effective fluctuating field in $\boldsymbol{k}$ space. The colored arrows mark the direction of $\boldsymbol{B}_{\|}$. Right: Spin orientation of eigenvectors for (a) linear Rashba, (b) Dresselhaus, and (c) cubic Dresselhaus.

scattering events, averaging out the effective magnetic field of SOC. As the magnetic field is tilted, the cyclotron frequency decreases, suppressing motional narrowing. Suppression of motional narrowing results in an increase of decoherence, increasing $T_{2}^{-1}$.

In the linear SOC limit with both Rashba and Dresselhaus coupling, we observe that the anisotropy is remarkable, with $T_{2}^{-1}$ differing by an order of magnitude at $\theta=\pi / 4$ $\left(\sin ^{2} \theta=0.5\right)$ for two different crystallographic directions of $B_{\|}$(magenta, $\varphi=-\pi / 4$ and green, $\varphi=\pi / 4$ ). The solid 8shaped curve in the left inset corresponds to the magnitude of the fluctuating (SOC) field in the linear limit (note that it is maximal along $\varphi=\pi / 4$ and minimal along $\varphi=-\pi / 4$ ), while the black arrows mark the corresponding direction of this effective field in $k$-space. The colored arrows represent the $B_{\|}$ direction in the crystallographic plane for the five chosen directions, $\varphi=0, \pm \pi / 8, \pm \pi / 4$. As moderate cubic terms are included, the anisotropy is enhanced (thin green curve). The broken green curve corresponds to the limit $\Gamma_{\alpha, \beta} \gg \chi \sim 1.5$, when the cubic terms dominate anisotropy.

\section{B. Relation to experiments}

We now analyze existing experimental data, [9, 31, 35, 36], by taking into account the angular anisotropies introduced in Eq. 21] and employing the relative couplings $\Gamma_{\alpha, \beta}, \chi$ as fitting parameters. We focus on experimental results in $\mathrm{Si} / \mathrm{SiGe}$ heterostructures, since there exist a number of experiments with unexplained anisotropy. In these samples, the 2-DEG is formed within a $l \mathrm{~nm}$ thick strained Si layer grown on a strainrelaxed $\mathrm{Si}_{1-x} \mathrm{Ge}_{x}, x=.25-.35$. We note that the main parameter characterizing the samples is $\tau_{k}$, obtained from electron mobility data on the pertaining references. In these experiments, the scattering time shall be compared with any other time scales for typical experimental fields ( $1 \mathrm{~T})$. The cyclotron time is $\left(\tau_{c}=2 \pi / \omega_{c}\right) \sim 10 \mathrm{ps}$ (note that this one increases with $\theta$ ), and the "Larmor time" is $\left(\tau_{l}=2 \pi / \omega_{l}\right) \sim 10^{2}$ ps. Further details on each sample are given on table $\Pi$.

TABLE II. Transport parameters for the experimental data considered in this work: momentum scattering time, $\tau_{k}$, average value of $g$-factor, width of Si $Q W, l$, and carrier concentration $n_{e}$.

\begin{tabular}{|c||c|c|c|c|}
\hline Sample & $\tau_{k}[\mathrm{ps}]$ & $g$-factor & $\mathrm{Si} \mathrm{QW} l[\mathrm{~nm}]$ & $n_{e}\left[\times 10^{11} \mathrm{~cm}^{-2}\right]$ \\
\hline Ref. [35] & 10.2 & 2.0005 & 15 & 1.0 \\
\hline Ref. [36] & 10 & 2.0005 & 20 & 3.0 \\
\hline Ref. [8] & 10.2 & - & 20 & 3.0 \\
\hline UW-30903 & 9.4 & 2.0005 & 10 & 4.3 \\
\hline UW-030827 & 9.7 & 2.0013 & 10 & 4.8 \\
\hline IBM-01 & 4.3 & 2.00013 & 8 & 4.0 \\
\hline UW-31203 & 1.8 & 2.0003 & 10 & 2.6 \\
\hline UW-31124 & 6.9 & 2.0012 & 10 & 4.7 \\
\hline UW-31121 & 5.0 & 2.0013 & 10 & 5.4 \\
\hline \hline
\end{tabular}

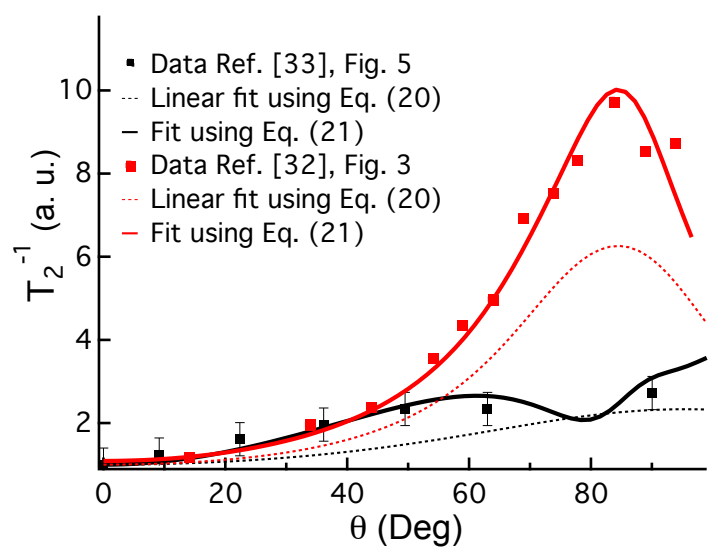

FIG. 3. Experimental data (squares), theoretical fits including cubic terms (solid curves), and fits including linear SOC terms (broken curves). Black: Ref. [35], fitted with $\chi=0.8$ and $\Gamma_{\beta}=0.2$ Red: Ref. [36], fitted with $\chi=10$ and $\Gamma_{\beta}=2$.

Fig. 3 shows extracted data from Refs. [35] (black) and [36] (red), respectively. The solid lines are theoretical fits using Eq. 21], whereas the broken ones are fits including only linear SOC terms, as in Eq. 20]. For the former case, a small cubic term $\Gamma_{\beta}=0.2$ allows us to reproduce the experimental data of Graeff et al., with $\beta=.8 \alpha$. The black lines, however, fit the data of Wilamowski et al. with $\chi=10$ and $\Gamma_{\beta}=2$, coinciding with the parameters used by Tyryshkin $e t$ al. [8] (not shown). This would imply $\Gamma_{\beta}=.2 \beta$ and $\beta=10 \alpha$, that is, a system dominated by linear Rashba and with a sizable cubic contribution. One data point from Jantsch et al. would be consistent with $\chi=1.5$ and a large cubic term, $\Gamma_{\beta}=7.5$. We stress that we do not attempt to extract the relative coupling strengths, $\chi, \Gamma_{\alpha, \beta}$, as different combinations of the three parameters could be consistent with the data. A more reliable determination of the parameters would require 
$\varphi$-resolved measurements. However, absence of cubic terms in the SOC would render impossible a fit to the data analyzed so far, for DP-relaxation.

Now we focus on the six samples presented by Truitt et al. [31]. The experimental data are the dots of Fig. 4, which we next attempt to fit using Eq. 21) (solid lines). Once again, a fit that includes only one linear term for the SOC, $\chi=0$ (i. e., only one source of linear SOC), failed to reproduce most of the experimental results (red curves), as it was attempted by Truitt et al.. Only the narrower sample (IBM-01) agreed to this DP, linear-in momentum fit. This is consistent with the established picture where SOC strengths are sample dependent and very sensitive to well widths [20, 30]. The blue lines correspond to the up-bound limit of anisotropy by including both Rashba and Dresselhaus SOC, which is still far from reasonable. Including both linear terms and cubic (black curves) contributions of SOC allows to reproduce most of the experimental data. However, we need make a distinction between the left and right panels of Fig. 4. The former present larger mobility and a cubic fit seem to agree very well with the data, as those in Fig. 3, whereas the latter ones have a lower mobility and fail to reproduce DP relaxation, especially for sample 'UW-31203'.

In the low mobility limit, tilted fields enhance surface roughness scattering, resulting from the squeezing of the $2 \mathrm{D}$ well in the growth direction [32]. Scattering processes would then lead to anisotropic Elliot-Yafet (EY) mechanism for relaxation [33], which is proportional to the scattering time, $T_{2}^{-1}=\alpha_{\mathrm{EY}}(\theta) \tau_{k}^{-1}$. Here the EY coefficient $\alpha_{\mathrm{EY}}$ depends on the amount of admixture of different spin states and reflects the probability that a spin-flip process occurs in a momentum scattering event. This effect has been already experimentally observed in the lower mobility samples [34-36]. A linear dependence of dephasing on a parallel magnetic field has indeed been attributed to an inhomogeneous dephasing in the EY limit [34, 37] due to a variation $\Delta g$ of the $g$-factor, $\alpha_{E Y} \sim \Delta g \mu_{B} B \sin \theta / 2 \hbar$. We identify this source with our discrepancy with the theoretical fit on 'UW-21203'. The inset of the upper right panel of Fig. 2 represents the discrepancy of the DP fit with the experimental data, showing a linear dependency for low tilted angles, which is well fitted with $\Delta g \simeq 10^{-4}$. This is in excellent agreement with the experimental value [35, 38]. It is worth noting that ' $U W-31203$ ' data presents a pronounced 'cusp-like' behavior (see upperright panel of Fig. 4), enforcing our arguments. The same argumentation may be applied to samples uw-31124 and uw31121 , where a cusp appears evident. Again, $\varphi$-resolved measurements would yield the answer.

Note added: During the final stage of this manuscript preparation, Song et al. reported on EY-mechanism in Si QWs. This mechanism is enhanced in lower mobility samples [40]. Their research enforces the last argument presented for the lower mobility samples of Fig. 4

\section{CONCLUSIONS}

In summary, we derived an expression for the angular dependency of the decoherence time in a quantum well, where the choice of the appropriate coordinate system is crucial. We find that the interplay of the Rashba and Dresselhaus SOC is reflected in the anisotropy of $T_{2}$, suggesting a practical scheme to determine their relative strength in the linear-inmomentum limit. Although frequently ignored, we predict strongly enhanced DP processes due to the cubic terms of the SOC. Experimental data owing to high mobility samples agree very well with our theoretical assumptions. Additional ElliotYafet mechanisms may explain further discrepancies in samples with lower mobility.

Acknowledgments. We are grateful to A. Chudnovskiy and R. Joynt for enlightening discussions. This work was supported by the Deutsche Forschungsgemeinschaft via the Graduiertenkolleg 1286 "Functional Metal-Semiconductor Hybrid Systems".

\section{Appendix A: SIA and BIA in magnetic coordinates}

In order to express the Hamiltonian of Eq. 27 in the magnetic coordinates, we employ the Euler-Rodrigues formulation, for which the rotation matrix of angle $\theta$ around an axis defined by $\hat{n}=-\hat{i} \sin \varphi+\hat{j} \cos \varphi$ (see Fig. 1) is given by:

$$
R_{\hat{n}}^{\theta}=\left(\begin{array}{ccc}
1-\cos ^{2} \varphi(1-\cos \theta) & -\cos \varphi \sin \varphi(1-\cos \theta) & \cos \varphi \sin \theta \\
-\cos \varphi \sin \varphi(1-\cos \theta) & 1-\sin ^{2} \varphi(1-\cos \theta) & \sin \varphi \sin \theta \\
-\cos \varphi \sin \theta & -\sin \varphi \sin \theta & \cos \theta
\end{array}\right) \equiv\left(\begin{array}{ccc}
F_{1} & -F_{3} & F_{4} \\
-F_{3} & F_{2} & F_{5} \\
-F_{4} & -F_{5} & F_{6}
\end{array}\right)
$$

Without loosing generality, we focus on samples grown along $\hat{k}=[001]$ direction, and set the other main crystallographic directions as $\hat{i}\|[100], \hat{j}\|[010]$. In magnetic axes, we thus have:

$$
\begin{aligned}
& \hat{i}_{x, y, z}=R\left(\theta, \hat{n}^{\prime}\right) \hat{i}=\left(F_{1},-F_{3},-F_{4}\right) ; \\
& \hat{j}_{x, y, z}=R\left(\theta, \hat{n}^{\prime}\right) \hat{j}=\left(-F_{3}, F_{2},-F_{5}\right) ; \\
& \hat{k}_{x, y, z}=R\left(\theta, \hat{n}^{\prime}\right) \hat{k}=\left(F_{4}, F_{5}, F_{6}\right) .
\end{aligned}
$$

On the other hand, we have that the direction of $\hat{k}$ changes in time, and hence we define the angle $\phi_{k}(t)$ :

$$
\begin{aligned}
\vec{k} & =k_{F}\left(\hat{i} \cos \phi_{k}+\hat{j} \sin \phi_{k}\right)=k_{F}\left[\left(F_{1} \cos \phi_{k}-F_{3} \sin \phi_{k}\right) \hat{x}+\right. \\
& \left.+\left(-F_{3} \cos \phi_{k}+F_{2} \sin \phi_{k}\right) \hat{y}-\left(F_{4} \cos \phi_{k}+F_{5} \sin \phi_{k}\right) \hat{z}\right]
\end{aligned}
$$



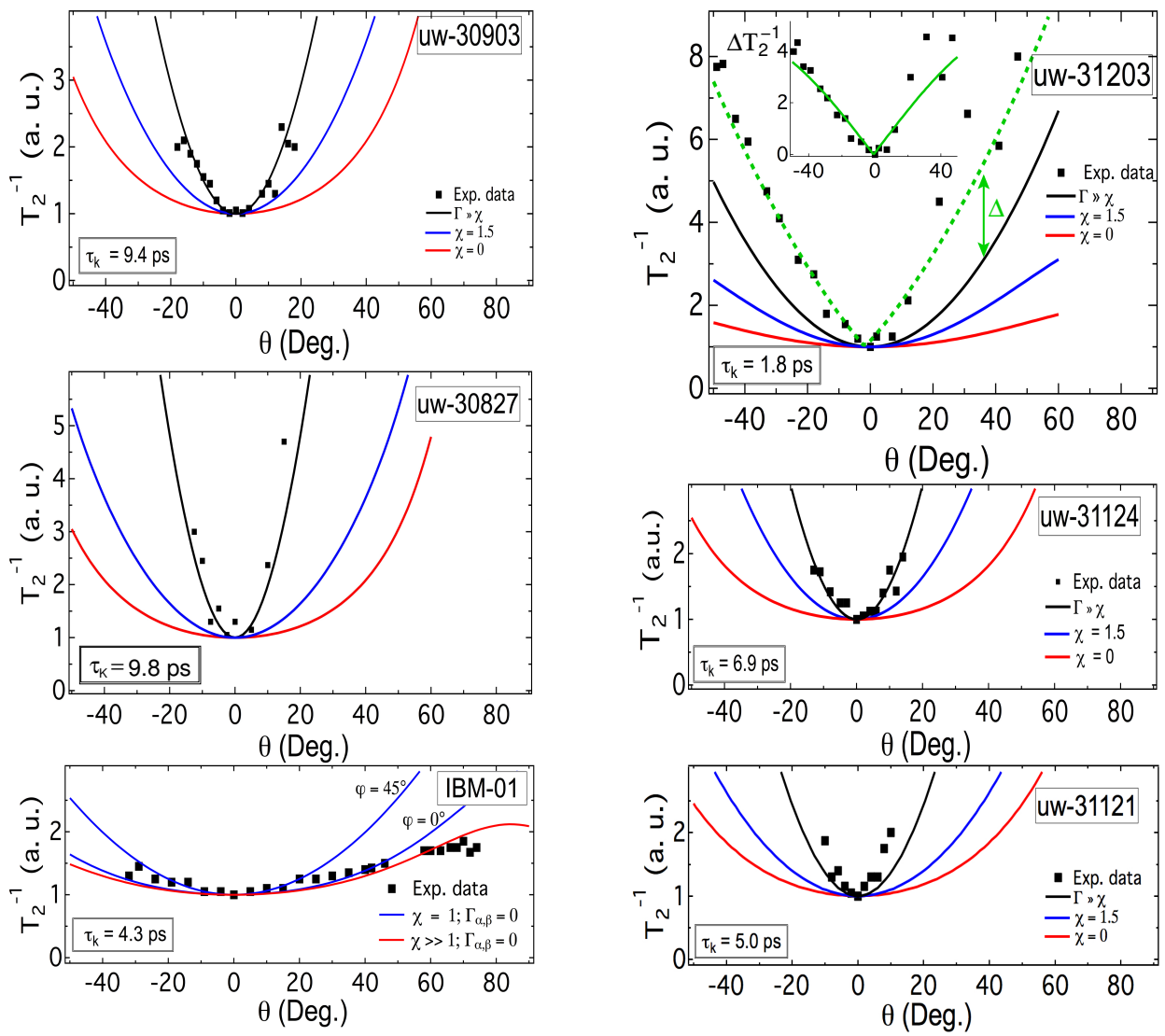

FIG. 4. Experimental data (squares) and theoretical fits (solid curves), including Rashba SOC as the only source of anisotropy (red), both Rashba and Dresselhaus linear terms SOC (blue) and cubic terms (black). Green broken line: additional EY mechanism could be the responsible for the observed anisotropy.

Using Eq. (A1) and (A2) into the first term of Eq. (2), we obtain the expression of the Rashba Hamiltonian in magnetic coordinates:

$$
\begin{aligned}
H_{R}=\alpha k_{F}\{ & \sigma_{x}\left[\left(F_{4} F_{5}-F_{3} F_{6}\right) \cos \phi_{k}+\left(F_{6} F_{2}+F_{5}^{2}\right) \sin \phi_{k}\right]- \\
& \sigma_{y}\left[\left(F_{4}^{2}+F_{1} F_{6}\right) \cos \phi_{k}+\left(F_{4} F_{5}-F_{6} F_{3}\right) \sin \phi_{k}\right]+ \\
& \left.\sigma_{z}\left[\left(F_{1} F_{5}+F_{3} F_{4}\right) \cos \phi_{k}-\left(F_{3} F_{5}+F_{2} F_{4}\right) \sin \phi_{k}\right]\right\} .
\end{aligned}
$$

For small $\theta$ we have $\phi_{k}(t) \simeq \omega_{c} t$ and $\omega_{c} \simeq\left(e B / m^{*} c\right) \cos \theta$ being the cyclotron frequency, and we obtain:

$$
H_{\mathrm{R}}=\vec{\sigma} \vec{B}_{R}^{\text {eff }}, \quad \vec{B}_{R}^{\text {eff }}=\alpha k_{F}\left(\hat{i} \sin \omega_{c} t-\hat{j} \cos \omega_{c} t\right)=\operatorname{Re}\left\{e^{i \omega_{c} t} \vec{Z}_{R}\right\},
$$

which is an effective field that rotates in the plane with direction $\omega_{c} \hat{k}$. Expressed in the magnetic axis, we may use $\mathrm{A} 3$ to get:

$$
\vec{Z}_{R}(\theta, \varphi)=\alpha k_{F} \sum_{q}\left(f_{q R}^{c}(\theta, \varphi)-i f_{q R}^{s}(\theta, \varphi)\right) \hat{q}, \quad q=x, y, z
$$

with

$$
\begin{array}{ll}
f_{x R}^{c}=\cos \varphi \sin \varphi(1-\cos \theta) ; & f_{x R}^{s}=\sin ^{2} \varphi+\cos ^{2} \varphi \cos \theta \\
f_{y R}^{c}=\cos ^{2} \varphi+\sin ^{2} \varphi \cos \theta ; & f_{y R}^{s}=\cos \varphi \sin \varphi(1-\cos \theta) \\
f_{z R}^{c}=\sin \varphi \sin \theta ; & f_{z R}^{s}=-\sin \varphi \sin \theta
\end{array}
$$

We consider next the Dresselhaus term for the lowest subband, assuming zero average momentum along the growth direction, $\langle\vec{k} \cdot \hat{k}\rangle=0$ :

$$
H_{D}=\beta k_{F}\left(\hat{\sigma}_{i} k_{i}-\hat{\sigma}_{j} k_{j}\right)=\beta k_{F}\left(\hat{\sigma}_{i} \cos \phi_{k}-\hat{\sigma}_{j} \sin \phi_{k}\right),
$$

where we have defined $\beta=\gamma_{D}\left\langle\left(k_{j}^{2}-k_{k}^{2}\right)\right\rangle$, and we have set: $\left\langle k_{j}^{2}\right\rangle=\left\langle k_{i}^{2}\right\rangle$. As before, we express the Dresselhaus Hamiltonian in the magnetic axes, for which we transform $\hat{\sigma}_{i, j}$ to get the rotated $\hat{\sigma}_{x, y, z}$ :

$$
\begin{aligned}
& \hat{\sigma}_{i} \rightarrow R\left(\theta, \hat{n}^{\prime}\right) \hat{\sigma}_{i}=F_{1} \hat{\sigma}_{x}-F_{3} \hat{\sigma}_{y}-F_{4} \hat{\sigma}_{z} \\
& \hat{\sigma}_{j} \rightarrow R\left(\theta, \hat{n}^{\prime}\right) \hat{\sigma}_{j}=-F_{3} \hat{\sigma}_{x}+F_{2} \hat{\sigma}_{y}-F_{5} \hat{\sigma}_{z},
\end{aligned}
$$

yielding:

$$
\begin{gathered}
H_{D}=\beta k_{F}\left[\hat{\sigma}_{x}\left(F_{1} \cos \phi_{k}+F_{3} \sin \phi_{k}\right)-\hat{\sigma}_{y}\left(F_{3} \cos \phi_{k}+F_{2} \sin \phi_{k}\right)+\right. \\
\left.+\hat{\sigma}_{z}\left(-F_{4} \cos \phi_{k}+F_{5} \sin \phi_{k}\right)\right] .
\end{gathered}
$$

The direction of the associated effective field rotates antiparallel to $B_{0}$,

$H_{\mathrm{D}}=\vec{\sigma} \vec{B}_{D}^{\text {eff }}, \quad \vec{B}_{D}^{\text {eff }}=\beta k_{F}\left(\hat{i} \cos \omega_{c} t-\hat{j} \sin \omega_{c} t\right)=\operatorname{Re}\left\{e^{-i \omega_{c} t} \vec{Z}_{D}\right\}$,

which is an effective field that rotates in the plane with direction $-\omega_{c} \hat{k}$, hence in opposite direction to the Rashba field. In terms of the magnetic axis, we use (A7) to get:

$$
\vec{Z}_{D}(\theta, \varphi)=\beta k_{F}\left(f_{q D}^{c}-i f_{q D}^{s}\right) \hat{q}, \quad q=x, y, z
$$


with

$f_{x D}^{c}=1-\cos ^{2} \varphi(1-\cos \theta) ; \quad f_{x D}^{s}=\cos \varphi \sin \varphi(1-\cos \theta) ;$

$f_{y D}^{c}=-\cos \varphi \sin \varphi(1-\cos \theta) ; \quad f_{y D}^{s}=\sin ^{2} \varphi(1-\cos \theta)-1 ;$

$f_{z D}^{c} \quad=-\cos \varphi \sin \theta ; \quad f_{z D}^{s}=\sin \varphi \sin \theta$.

As we can see $H^{\prime}$ has terms in $\sigma_{z}$, which are proportional to $\sin \theta$ and contribute to both decoherence and relaxation processes.

SIA and BIA terms of $n$-order in momentum may be obtained using this procedure, only that the "effective magnetic fields' rotate in time at a higher frequency, which is $n$-times faster than those for the linear terms. For the cubic terms, we have:

$$
\begin{aligned}
& \vec{B}_{3 R}^{\text {eff }}=\alpha_{3}\left(\vec{k}^{3}\right)\left(\hat{i} \sin \left(3 \omega_{c} t\right)-\hat{j} \cos \left(3 \omega_{c} t\right)\right), \\
& \vec{B}_{3 D}^{\text {eff }}=\beta_{3}\left(\vec{k}^{3}\right)\left(\hat{i} \cos \left(3 \omega_{c} t\right)-\hat{j} \sin \left(3 \omega_{c} t\right)\right) .
\end{aligned}
$$

yielding the components listed in table 1 .

\section{Appendix B: Bloch equations}

To evaluate the trace of the double commutator on second term of Eq. [11, we sum over all possible states and transform back to Schrödinger representation using Eq (8). Adopting the summation convention,

$$
\begin{aligned}
\operatorname{Tr} & \left\{\int_{0}^{\infty}\left[\left[\sigma_{i}^{*}(t), \sigma_{q}^{*}(t-\tau)\right], \sigma_{q^{\prime}}^{*}(t)\right] \sigma_{r}^{*}(t) u_{q q^{\prime}}(\tau) \mathrm{d} \tau\right\}= \\
& =n_{i}(t)\left\langle\alpha\left|\sigma_{i}\right| \beta\right\rangle\left\langle\beta\left|\sigma_{q}\right| \gamma\right\rangle\left\langle\gamma\left|\sigma_{q^{\prime}}\right| \delta\right\rangle\left\langle\delta\left|\sigma_{r}\right| \alpha\right\rangle b_{q q^{\prime}}\left(\omega_{\beta \gamma}\right)+ \\
& +n_{i}(t)\left\langle\alpha\left|\sigma_{q^{\prime}}\right| \beta\right\rangle\left\langle\beta\left|\sigma_{q}\right| \gamma\right\rangle\left\langle\gamma\left|\sigma_{i}\right| \delta\right\rangle\left\langle\delta\left|\sigma_{r}\right| \alpha\right\rangle b_{q q^{\prime}}\left(\omega_{\beta \gamma}\right)- \\
& -n_{i}(t)\left\langle\alpha\left|\sigma_{q}\right| \beta\right\rangle\left\langle\beta\left|\sigma_{i}\right| \gamma\right\rangle\left\langle\gamma\left|\sigma_{q^{\prime}}\right| \delta\right\rangle\left\langle\delta\left|\sigma_{r}\right| \alpha\right\rangle b_{q q^{\prime}}\left(\omega_{\alpha \beta}\right)- \\
& -n_{i}(t)\left\langle\alpha\left|\sigma_{q^{\prime}}\right| \beta\right\rangle\left\langle\beta\left|\sigma_{i}\right| \gamma\right\rangle\left\langle\gamma\left|\sigma_{q}\right| \delta\right\rangle\left\langle\delta\left|\sigma_{r}\right| \alpha\right\rangle b_{q q^{\prime}}\left(\omega_{\gamma \delta}\right)= \\
& =n_{i}(t) b_{q q^{\prime}}\left(\omega_{\alpha \beta}\right)\left\langle\alpha\left|\sigma_{q}\right| \beta\right\rangle\left\langle\beta\left|\left[\left[\sigma_{q^{\prime}}, \sigma_{r}\right], \sigma_{i}\right]\right| \alpha\right\rangle
\end{aligned}
$$

Using the identities (11):

$$
\left[\left[\sigma_{q^{\prime}}, \sigma_{r}\right], \sigma_{i}\right]=(2 i)^{2} \varepsilon_{q^{\prime} r l} \varepsilon_{l i k}=-4\left[\delta_{q^{\prime} i} \delta_{r k}-\delta_{q^{\prime} k} \delta_{r i}\right],
$$

Eq. (12) follows straightforward.

We now evaluate Eq. (12) for all three possible values of $r$. First, for $r=x$, we have:

$\frac{\mathrm{d} n_{x}(t)}{\mathrm{d} t}=\frac{2}{\hbar^{2}} n_{i}(t)\left(\delta_{q^{\prime} i} \delta_{x k}-\delta_{q^{\prime} k} \delta_{i x}\right)\left\langle\alpha\left|\sigma_{q}\right| \beta\right\rangle\left\langle\beta\left|\sigma_{k}\right| \alpha\right\rangle b_{q q^{\prime}}\left(\omega_{\alpha \beta}\right)$.

The Pauli matrices for spin $1 / 2$ read,

$$
\sigma_{x}=\left(\begin{array}{cc}
0 & 1 \\
1 & 0
\end{array}\right) ; \quad \sigma_{y}=\left(\begin{array}{cc}
0 & -i \\
i & 0
\end{array}\right) ; \quad \sigma_{z}=\left(\begin{array}{cc}
1 & 0 \\
0 & -1
\end{array}\right) ;
$$

and $|\alpha\rangle=\uparrow, \downarrow$ and $|\beta\rangle=\uparrow, \downarrow$. For the second factor on the right, there are two terms. The first one, has $k=x$, so $q^{\prime}=x, y, z$ and $q=x, y$, giving:

$$
\begin{aligned}
& \frac{2}{\hbar^{2}} n_{q^{\prime}}(t)\left\langle\alpha\left|\sigma_{q}\right| \beta\right\rangle\left\langle\beta\left|\sigma_{x}\right| \alpha\right\rangle b_{q q^{\prime}}\left(\omega_{\alpha \beta}\right)= \\
= & \frac{2}{\hbar^{2}}\left\{n_{x}\left[b_{x x}\left(\omega_{l}\right)+b_{x x}\left(-\omega_{l}\right)-i b_{y x}\left(\omega_{l}\right)+i b_{y x}\left(-\omega_{l}\right)\right]+\right. \\
& +n_{y}\left[b_{x y}\left(\omega_{l}\right)+b_{x y}\left(-\omega_{l}\right)-i b_{y y}\left(\omega_{l}\right)+i b_{y y}\left(-\omega_{l}\right)\right]+ \\
& +n_{z}\left[b_{x z}\left(\omega_{l}\right)+b_{x z}\left(-\omega_{l}\right)-i b_{y z}\left(\left(\omega_{l}\right)+i b_{y z}\left(-\omega_{l}\right)\right]\right\},
\end{aligned}
$$

and for the second term, $i=x$ while $k=q^{\prime}$, giving:

$$
\begin{aligned}
& -\frac{2}{\hbar^{2}} n_{x}(t)\left\langle\alpha\left|\sigma_{q}\right| \beta\right\rangle\left\langle\beta\left|\sigma_{q^{\prime}}\right| \alpha\right\rangle b_{q q^{\prime}}\left(\omega_{\alpha \beta}\right)= \\
& \quad-\frac{2}{\hbar^{2}}\left\{n _ { x } \left[b_{x x}\left(\omega_{l}\right)+b_{x x}\left(-\omega_{l}\right)+i b_{x y}\left(\omega_{l}\right)-i b_{x y}\left(-\omega_{l}\right)-\right.\right. \\
& \left.\left.\quad-i b_{y x}\left(\omega_{l}\right)+i b_{y x}\left(-\omega_{l}\right)+b_{y y}\left(\omega_{l}\right)+b_{y y}\left(-\omega_{l}\right)+2 b_{z z}(0)\right]\right\} .
\end{aligned}
$$

On what follows, we will assume $b_{q q}(\omega)=b_{q q}(-\omega)$. Inserting these last two equations into $(\overline{\mathrm{B} 2})$, we get:

$$
\begin{aligned}
\frac{\mathrm{d} n_{x}(t)}{\mathrm{d} t}= & -\frac{4}{\hbar^{2}}\left\{n_{x}\left[b_{y y}\left(\omega_{l}\right)+b_{z z}(0)\right]-n_{y}\left[b_{x y}\left(\omega_{l}\right)\right]-\right. \\
& \left.-n_{z}\left[b_{x z}\left(\omega_{l}\right)\right]\right\} .
\end{aligned}
$$

We now evaluate Eq. (12) for $r=y$,

$\frac{\mathrm{d} n_{y}(t)}{\mathrm{d} t}=\frac{2}{\hbar^{2}} n_{i}(t)\left(\delta_{q^{\prime} i} \delta_{y k}-\delta_{q^{\prime} k} \delta_{i y}\right)\left\langle\alpha\left|\sigma_{q}\right| \beta\right\rangle\left\langle\beta\left|\sigma_{k}\right| \alpha\right\rangle b_{q q^{\prime}}\left(\omega_{\alpha \beta}\right)$.

For the second factor on the right, there are two terms. The first one, has $k=y$, so $q^{\prime}=x, y, z$ and $q=x, y$, giving:

$$
\begin{aligned}
& \frac{2}{\hbar^{2}} n_{q^{\prime}}(t)\left\langle\alpha\left|\sigma_{q}\right| \beta\right\rangle\left\langle\beta\left|\sigma_{y}\right| \alpha\right\rangle b_{q q^{\prime}}\left(\omega_{\alpha \beta}\right)= \\
& \frac{2}{\hbar^{2}}\left\{n_{x}\left[i b_{x x}\left(\omega_{l}\right)-i b_{x x}\left(-\omega_{l}\right)+b_{y x}\left(\omega_{l}\right)+b_{y x}\left(-\omega_{l}\right)\right]+\right. \\
+ & n_{y}\left[i b_{x y}\left(\omega_{l}\right)-i b_{x y}\left(-\omega_{l}\right)+b_{y y}\left(\omega_{l}\right)+b_{y y}\left(-\omega_{l}\right)\right]+ \\
+ & n_{z}\left[i b_{x z}\left(\omega_{l}\right)-i b_{x z}\left(-\omega_{l}\right)+b_{y z}\left(\left(\omega_{l}\right)+b_{y z}\left(-\omega_{l}\right)\right]\right\},
\end{aligned}
$$

and for the second term, $i=y$ while $k=q^{\prime}$, giving:

$$
\begin{aligned}
& -\frac{2}{\hbar^{2}} n_{y}(t)\left\langle\alpha\left|\sigma_{q}\right| \beta\right\rangle\left\langle\beta\left|\sigma_{q^{\prime}}\right| \alpha\right\rangle b_{q q^{\prime}}\left(\omega_{\alpha \beta}\right)= \\
& -\frac{2}{\hbar^{2}}\left\{n _ { y } \left[b_{x x}\left(\omega_{l}\right)+b_{x x}\left(-\omega_{l}\right)+i b_{x y}\left(\omega_{l}\right)-i b_{x y}\left(-\omega_{l}\right)-\right.\right. \\
& \left.\left.\quad-i b_{y x}\left(\omega_{l}\right)+i b_{y x}\left(-\omega_{l}\right)+b_{y y}\left(\omega_{l}\right)+b_{y y}\left(-\omega_{l}\right)+2 b_{z z}(0)\right]\right\} .
\end{aligned}
$$

Collecting these last results, we get:

$$
\begin{aligned}
\frac{\mathrm{d} n_{y}(t)}{\mathrm{d} t} & =\frac{4}{\hbar^{2}}\left\{n_{x}\left[b_{y x}\left(\omega_{l}\right)\right]-n_{y}\left[b_{x x}\left(\omega_{l}\right)+2 b_{z z}(0)\right]+\right. \\
& +n_{z}\left[b_{y z}\left(\left(\omega_{l}\right)\right]\right\} .
\end{aligned}
$$

Finally, for $r=z$ in $(12)$, we get:

$\frac{\mathrm{d} n_{z}(t)}{\mathrm{d} t}=-\frac{2}{\hbar^{2}} n_{i}(t)\left(\delta_{q^{\prime} i} \delta_{z k}-\delta_{q^{\prime} k} \delta_{i z}\right)\left\langle\alpha\left|\sigma_{q}\right| \beta\right\rangle\left\langle\beta\left|\sigma_{k}\right| \alpha\right\rangle b_{q q^{\prime}}\left(\omega_{\alpha \beta}\right)$.

The first term on the right has $k=z$, and thus only $q=z$ contributes, with $q^{\prime}=i=x, y, z$, whereas the second term on the right has $i=z$ with $q, q^{\prime}=x, y, z$,

$$
\begin{aligned}
\frac{\mathrm{d} n_{z}(t)}{\mathrm{d} t} & =\frac{4}{\hbar^{2}}\left\{n_{x}(t) b_{z x}(0)+n_{y}(t) b_{z y}(0)+n_{z} b_{z z}(0)\right. \\
& \left.-n_{z}(t)\left[b_{x x}\left(\omega_{l}\right)+b_{y y}\left(\omega_{l}\right)+b_{z z}(0)\right] .\right\}
\end{aligned}
$$

Equations B3 $|\mathrm{B} 4| \mathrm{B} 5$ ) are indeed the Bloch equations in (14). 
[1] F. A. Zwanenburg, et al., Rev. Mod. Phys. 85

[2] J. L. Morton, D. R. McCamey, M. A. Eriksson, and S. A. Lyon. Nature 479, 345-353 (2011).

[3] R. Sanchez and G. Platero, Phys. Rev. B 87, 081305(R) (2013).

[4] S. Datta, and B. Das, Appl. Phys. Lett. 56, 665 (1990).

[5] I. Žutić, J. Fabian, and S. Das Sarma, Rev. Mod. Phys. 76, 323 (2004).

[6] C. Tahan, and R. Joynt, Phys. Rev. B 89075302 (2014).

[7] E. Kawakami, et al., Nature Nanotech. 9666 (2014).

[8] A. M. Tyryshkin, S. A. Lyon, W. Jantsch, and F. Schäffler, Phys. Rev. Lett. 94, 126802 (2005);

[9] S. Shankar, A. M. Tyryshkin, J. He, and S. A. Lyon, Phys. Rev. B 82, 195323 (2010); A. Morello et al., Nature 467, 687 (2010).

[10] C. H. Wong, M. A. Eriksson, S. N. Coppersmith, and M. Friesen, Phys. Rev. B 92, 045403 (2015).

[11] N. Rohling, M. Russ, and G. Burkard, Phys. Rev. Lett. 113, 176801 (2014).

[12] M. Veldhorst et al., Nature 526, 410 (2015)

[13] D. Loss, and D. P. DiVincenzo, Phys. Rev. A 57, 120-126 (1998); R. De Sousa, and S. Das Sarma, Phys. Rev. B 67, 033301 (2003).

[14] H. Sanada, Y. Kunihashi, H. Gotoh, K. Onomitsu, M. Kohda, J. Nitta, P. V. Santos, and T. Sogawa, Nature Phys. 9, 280-283 (2013).

[15] F. H. L. Koppens et al., Nature 442, 766 (2006).

[16] G. Dresselhaus, Phys. Rev. 100, 580586 (1955); R. Wrinkler, Spin-Orbit Coupling Effects in Two-Dimensional Electron and Hole System. Springer-Verlag, Berlin-Heidelberg-New York, 2003.

[17] I. V. Tokatly, and E. Ya. Sherman, Phys. Rev. B 82, 161305(R) (2010); M. M. Glazov, E. Ya. Sherman, and V. K. Dugaev, 42 2157 (2010).

[18] L. Vervoort and P. Voisin, Phys. Rev. B 56, 12744 (1997).

[19] Y. B. Lyanda-Geller, adn A. D. Mirlin, Phys. Rev. Lett. 72, 1894 (1994).

[20] M. Prada, G. Klimeck, R. Joynt, New J. Phys. 13, 013009 (2011).

[21] M. O. Nestoklon, L. E. Golub, E. L. Ivchenko, Phys. Rev. B 73, 235334 (2006).

[22] S. D. Ganichev, and L. E. Golub, Phys. Stat. Sol. B, 2511801 (2014).
[23] X. Cartoixà, L.-W. Wang, D.Z.-Y. Ting, and Y.-C. Chang, Phys. Rev. B 73, 205341 (2006); W. Yang and K. Chang, Phys. Rev. B 74, 193314 (2006); H. Nakamura, T. Koga and T. Kimura, Phys. Rev. Lett. 108, 206601(2012); R. Moriya et al., Phys. Rev. Lett. 113, 086601 (2014).

[24] C. P. Schlister, Principles of Magnetic Resonance, Springer Series in Solid-State Sciences, New York, (1963).

[25] A. Abragam, The Principles of Nuclear Magnetism, Oxford University Press, (2002).

[26] M. I. D’Yakonov and V. I. Perel'. Sov. Phys. Solid State 13:3023, 1971.

[27] F. Li, Y. V. Pershin, V. A. Slipko, and N. A. Sinitsyn, Phys. Rev. Lett. 111, 067201 (2013).

[28] A. G. Redfield, IBM J. Res. Dev. 1, 19 (1957).

[29] M. M. Glazov, Phys. Rev. B 70, 195314 (2004).

[30] M. Kohda et al., Phys. Rev. B 86, 081306(R) (2012).

[31] Electron spin coherence in $\mathrm{Si} / \mathrm{SiGe}$ quantum wells, J. L. Truitt, et al., cond-mat/0411735 Topics in Applied Physics Series 115, ed. M. Fanciulli (Springer, 2008).

[32] D. A. Syphers, J. E. Furneaux, Solid State Comm. 65, 15131515 (1988); T. Ando, A. B. Fowler, F. Stern, Rev. Mod. Phys. 54, 437-672 (1982).

[33] N. S. Averkiev, L. E. Golub, and M. Willander, J. Phys: Condens. Matter 14, R271-R283 (2002); S. D. Ganichev et al., Phys. Rev. B 68, 081302(R) (2003).

[34] M. Studer, S. Schön, K. Ensslin, and G. Salis, Phys. Rev. B 79, 045302 (2009).

[35] C. F. O. Graeff, M. S. Brandt, M. Stutzmann, M. Holzmann, G. Abstreiter, and F. Schäffler, Phys. Rev. B 59, 13242 (1999).

[36] Z. Wilamowski and W. Jantsch. Phys. Rev. B 69 035328, 2004.

[37] J. M. Kikkawa, and D. D. Awschalom, Phys. Rev. Lett. 80, 4313 (1998).

[38] Z. Wilamowski, W. Jantsch, H. Malissa, and U. Rössler, Phys. Rev. B 66, 195315 (2002).

[39] W. Jantsch, Z. Wilamowski, N. Sandersfeld, M. Mühlberger, and F. Schäffler, Physica E 13, 504 (2002).

[40] Y. Song, adn S. Das Sarma, arXiv:1606.09578 [cond-mat.meshall] 